\title{
SUPERVISIÓN DE ENSAYOS CLÍNICOS EN HOSPITALES DEL SEGURO SOCIAL DE SALUD DEL PERÚ: ENFOQUE ADMINISTRATIVO Y REGULATORIO
}

\author{
Jorge A. García-Mostajo, ${ }^{1, a}$, Fulton P. Rivera1,2,b, Violeta Alvarez-Arroyo, ${ }^{1, c}$, \\ Manuel Catacora-Villasante ${ }^{1,3, \mathrm{~d} *}$, Joshi Acosta $^{1, \mathrm{e} *}$
}

\begin{abstract}
RESUMEN
El objetivo del estudio fue describir las características de los ensayos clínicos (EC) supervisados por el Instituto de Evaluación de Tecnologías en Salud e Investigación en EsSalud entre el 2015 y 2018 y las principales observaciones de las supervisiones realizadas. Se realizó un estudio descriptivo de 82 ensayos clínicos supervisados entre 2015 y 2018. La mayoría de los ensayos clínicos fueron estudios de fase III (81,7\%), la vía de administración más frecuente de los productos de estudio fue oral $(47,6 \%)$ y la mayoría fueron patrocinados por la industria farmacéutica $(96,3 \%)$. Las observaciones más frecuentes fueron las relacionadas al contrato de estudio $(83,8 \%)$, al pago por concepto de overhead $(57,3 \%)$ y a la falta de documentos regulatorios $(47,6 \%)$. Estos hallazgos permiten la identificación de oportunidades de mejora en la regulación y gestión de la investigación.
\end{abstract}

Palabras clave: Ensayo Clínico; Seguridad Social; Perú (fuente: DeCS BIREME).

\section{SUPERVISION OF CLINICAL TRIALS IN SOCIAL HEALTH INSURANCE AT PERUVIAN HOSPITALS: ADMINISTRATIVE AND REGULATORY APPROACH}

\begin{abstract}
The objective of the study was to describe the characteristics of the Clinical Trials (CT) supervised by the Institute of Health Technology Assessment and Research carried out in EsSalud between 2015 and 2018 and the main observations of the supervisions completed. A descriptive study of 82 supervised clinical trials was conducted between 2015 and 2018 . Most of the clinical trials were phase III studies (81.7\%); the most frequent route of administration of the study products was oral $(47.6 \%)$, and most were sponsored by the pharmaceutical industry (96.3\%). The most frequent observations were those related to the study contract $(83.8 \%)$, overhead payment $(57.3 \%)$, and the lack of regulatory documents $(47.6 \%)$. These findings allow the identification of opportunities for improvement in research regulation and management.
\end{abstract}

Keywords: Clinical Trial; Social Security; Peru (source: MeSH NLM).

\section{INTRODUCCIÓN}

Los ensayos clínicos $(E C)$ logran avances en la atención médica y proveen información a los decisores en salud para identificar estrategias y tratamientos con mejores resultados ${ }^{(1)}$. Por ello, es necesario el cumplimiento de requisitos éticos y normativos que garanticen la protección a las personas y el valor de sus resultados.

En el Seguro Social de Salud del Perú (EsSalud), el Instituto de Evaluación de Tecnologías en Salud e Investigación
(IETSI) tiene la función de supervisar los EC que se desarrollan en los centros de investigación, de acuerdo con políticas y normas establecidas ${ }^{(2)}$, las que incluyen, entre otras, el Reglamento de Ensayos Clínicos ${ }^{(3)}$ y las propias de EsSalud (4). Estas supervisiones tienen un enfoque principalmente administrativo para lograr la mejora continua de los procesos dentro de una investigación clínica.

Existe escasa literatura sobre observaciones generadas durante la supervisión a EC y esta corresponde al reporte de patrocinadores y agencias reguladoras ${ }^{(5-7)}$. Sin embargo, en

\footnotetext{
Instituto de Evaluación de Tecnologías en Salud e Investigación - IETSI, EsSalud. Lima, Perú.

Universidad Científica del Sur. Lima, Perú.

Universidad Nacional Mayor de San Marcos. Lima, Perú.

Médico internista, magíster en Salud Pública; ${ }^{\mathrm{b}}$ tecnólogo médico, magíster en Microbiología; ${ }^{\mathrm{c}}$ médico cirujano; ${ }^{\mathrm{d}}$ médico psiquiatra; ${ }^{\mathrm{e}}$ médico especialista en Microbiología y Parasitología, doctora en Microbiología Clínica.

* El estudio se realizó cuando el autor fue trabajador del Instituto de Evaluación de Tecnologías en Salud e Investigación - IETSI, EsSalud.

Recibido: 24/05/2019 Aprobado: 06/11/2019 En línea: 03/12/2019
} 
el Perú no se disponen de investigaciones que describan las observaciones realizadas en supervisiones por parte de las instituciones donde se ejecutan los estudios.

El objetivo del estudio fue describir las características de los EC supervisados por el IETSI en EsSalud entre el 2015 y 2018 y las principales observaciones de las supervisiones realizadas.

\section{EL ESTUDIO}

Se realizó un estudio descriptivo de corte transversal que incluyó a todos los EC que fueron supervisados por el IETSI entre el 2015 y 2018 en los centros de investigación de EsSalud. Para 2019, EsSalud es una institución con una población afiliada de casi once millones ${ }^{(8)}$, aproximadamente un tercio de la población nacional, y alberga un número importante de centros de investigación que participan en EC.

En este periodo, la autoridad reguladora de EC en el Perú, el Instituto Nacional de Salud (INS) recibió y aprobó 196 estudios, de los cuales 92 (47\%) han tenido la participación de EsSalud con al menos un centro de investigación ${ }^{(9)}$. A pesar que EsSalud cuenta con 58 centros de investigación registrados y en condición de activos en el INS, los cuales corresponden a diversas especialidades médicas y están distribuidos en seis hospitales de Lima y seis de provincia, no todos se encuentran en funcionamiento y sólo en 22 de estos centros se ejecutan actualmente EC.

Los EC supervisados fueron elegidos teniendo en cuenta la fase de los mismos (seleccionando primero a los de fase más temprana), la inclusión de población que podría ser vulnerable y los que implicaban patologías de alto riesgo o costo (por ejemplo, enfermedades oncológicas o con terapia biológica). Asimismo, se priorizó la supervisión de estudios desarrollados en centros de investigación con mayor número de EC aprobados.

Las supervisiones son realizadas por personal especializado en el tema, estas son llevadas a acabo en los centros de investigación donde se ejecuta el estudio. El personal utiliza una ficha única, la cual incluía secciones relacionadas a la aprobación del estudio, documentación según buenas prácticas clínicas, contrato del estudio, pago por concepto de overhead, calibración de equipos, bioseguridad y otros ${ }^{(4)}$. Luego de cada supervisión se emitió un informe con las observaciones y recomendaciones, las mismas que podían ser dirigidas a cualquiera de las instancias involucradas en la ejecución de EC.

Para el estudio, se utilizó como fuente de datos las fichas de supervisión y los informes con las observaciones realizadas. Se registró la información obtenida de cada uno de los estudios en una base de datos en el programa

\section{MENSAJES CLAVE}

Motivación para realizar el estudio. Describir las características de ensayos clínicos ejecutados en EsSalud y el tipo de observaciones realizadas con mayor frecuencia durante las supervisiones.

Principales hallazgos. Entre el 2015 y el 2018 se hicieron 82 supervisiones a ensayos clínicos. Las observaciones más frecuentes fueron administrativas, relacionadas al contrato de estudio, al pago por concepto de overhead y a la falta de documentos regulatorios.

Implicancias. Estos hallazgos permiten la identificación de oportunidades de mejora en la regulación y gestión de la investigación desarrollada en EsSalud.

Microsoft Excel. Luego, se procedió con el análisis de la distribución de las diferentes variables y el cálculo de frecuencias relativas y absolutas.

En relación a los aspectos éticos, el presente estudio no constituye una investigación en seres humanos. La unidad de análisis no fueron sujetos de investigación, sino los EC supervisados, por lo que no requirió aprobación por un Comité de Ética en Investigación. En cuanto a los aspectos generales de los EC incluidos, la información es de acceso público en la página web del Registro de Ensayos Clínicos de los Institutos Nacionales de Salud de Estados Unidos (www.clinicaltrials.gov) ${ }^{(10)}$ o en la del INS (http:// www.ensayosclinicos-repec.ins.gob.pe/acerca-del-repec/ busqueda-de-ensayos-clinicos) ${ }^{(9)}$. No se presentan datos que permitan la identificación de los estudios supervisados e incluidos.

\section{HALLAZGOS}

Entre el 2015 y 2018 se supervisaron 82 EC en EsSalud, de los cuales $94 \%$ corresponden a cinco hospitales de Lima, incluyendo los tres de mayor complejidad y población asignada. Cinco supervisiones (6\%) fueron realizadas en hospitales de tres capitales de regiones.

En la Tabla 1 se describen las principales características de los EC supervisados. Durante los cuatro años, la mayoría $(81,7 \%)$ fueron de fase III y correspondían a las especialidades de oncología, reumatología, infectología y cardiología. En cuanto a la vía de administración del producto de investigación, la oral fue la más frecuente $(47,6 \%)$, seguida de la intravenosa $(32,9 \%)$. Todos fueron estudios multicéntricos y sólo dos no fueron multinacionales. La mayoría $(96,3 \%)$ fueron patrocinados por la industria farmacéutica, mientras que sólo tres $(3,7 \%)$ fueron patrocinados por una universidad privada peruana.

En relación a los eventos adversos serios (EAS), la frecuencia de EC supervisados con al menos un EAS fue variable en el tiempo: $58 \%$ el 2015 (7/12), 48\% el 2016 
Tabla 1. Características de los ensayos clínicos supervisados en hospitales del Seguro Social de Salud del Perú, EsSalud

\begin{tabular}{|c|c|c|c|c|c|}
\hline Característica & $\begin{array}{c}2015 \\
n\end{array}$ & $\begin{array}{c}2016 \\
n\end{array}$ & $\begin{array}{c}2017 \\
\mathrm{n}\end{array}$ & $\begin{array}{c}2018 \\
n\end{array}$ & $\begin{array}{l}\text { Total } \\
\text { N (\%) }\end{array}$ \\
\hline Ensayos clínicos supervisados & 12 & 25 & 25 & 20 & $82(100)$ \\
\hline \multicolumn{6}{|l|}{ Fase del estudio } \\
\hline Fase I & 0 & 0 & 0 & 1 & $1(1,2)$ \\
\hline Fase II & 1 & 2 & 4 & 2 & $9(11,0)$ \\
\hline Fase III & 11 & 21 & 20 & 15 & $67(81,7)$ \\
\hline Fase IV & 0 & 2 & 1 & 2 & $5(6,1)$ \\
\hline \multicolumn{6}{|l|}{ Cegamiento } \\
\hline Abierto & 4 & 6 & 13 & 7 & $30(36,6)$ \\
\hline Simple & 1 & 2 & 1 & 1 & $5(6,1)$ \\
\hline Doble & 7 & 17 & 11 & 12 & $47(57,3)$ \\
\hline \multicolumn{6}{|l|}{ Tipo de producto } \\
\hline Químico & 8 & 15 & 11 & 12 & $46(56,1)$ \\
\hline Biológico & 4 & 10 & 14 & 8 & $36(43,9)$ \\
\hline \multicolumn{6}{|l|}{ Vía de administración del producto } \\
\hline Oral & 6 & 11 & 9 & 13 & $39(47,6)$ \\
\hline Subcutánea & 0 & 3 & 2 & 3 & $8(9,8)$ \\
\hline Intravenosa & 5 & 9 & 10 & 3 & $27(32,9)$ \\
\hline Intramuscular & 1 & 2 & 1 & 0 & $4(4,9)$ \\
\hline Combinación de dos vías & 0 & 0 & 3 & 1 & $4(4,9)$ \\
\hline \multicolumn{6}{|l|}{ Patrocinador } \\
\hline Industria farmacéutica & 12 & 22 & 25 & 20 & $79(96,3)$ \\
\hline Institución académica & 0 & 3 & 0 & 0 & $3(3,7)$ \\
\hline \multicolumn{6}{|l|}{ Tipo de patología } \\
\hline Crónica & 8 & 19 & 22 & 11 & $60(73,2)$ \\
\hline Aguda & 3 & 5 & 2 & 5 & $15(18,3)$ \\
\hline Prevención & 1 & 1 & 1 & 4 & $7(8,5)$ \\
\hline Ensayos clínicos con eventos adversos serios & 7 & 12 & 25 & 9 & $53(65)$ \\
\hline
\end{tabular}

(12/25), $100 \%$ el $2017(25 / 25)$ y $45 \%(9 / 20)$ en el 2018. Durante todo el periodo se reportó un total de 263 EAS en los 82 estudios, de los cuales dos fueron catalogados como definitivamente relacionados con el producto de investigación, dos como probablemente relacionados y los demás como definitivamente no relacionados.

En la Tabla 2 se describen las principales observaciones realizadas durante las supervisiones. Si bien la variabilidad de observaciones fue amplia, se han agrupado para presentar las más frecuentes y relevantes, siendo la mayoría relacionadas al contrato de estudio (83,8\%), pago por concepto de overhead $(57,3 \%)$ y falta de documentos regulatorios $(47,6 \%)$.

\section{DISCUSIÓN}

Analizamos los documentos de las 82 supervisiones a EC realizadas en los hospitales de EsSalud entre el
2015 y 2018. De forma similar a un estudio previo (11), los EC supervisados correspondían a estudios de fase III $(82 \%)$ y eran patrocinados en su mayoría por la industria farmacéutica (96\%). Las observaciones más frecuentes encontradas en los EC supervisados fueron relacionadas al contrato de estudio (84\%), pago por concepto de overhead $(59 \%)$ y falta de documentos regulatorios (48\%).

En cuanto a las observaciones sobre el contrato de estudio, la normativa de EsSalud establecía desde el 2008 que este debía ser visado por la Gerencia del Hospital; sin embargo, en las supervisiones se identificó que esta no era una práctica frecuente. La limitada cantidad de estudios publicados sobre el tema y la variabilidad en la metodología y forma de categorizar y reportar los resultados dificultan las comparaciones, principalmente en lo correspondiente a aspectos normativos de cada institución, como es el caso de los contratos ${ }^{(12-14)}$. 
Tabla 2. Principales observaciones en los ensayos clínicos supervisados en los hospitales del Seguro Social de Salud del Perú entre el 2015 y el 2018 (N=82)

\begin{tabular}{lcc}
\hline $\begin{array}{l}\text { Categorías de } \\
\text { observaciones }\end{array}$ & $\begin{array}{c}\text { Número de } \\
\text { estudios en } \\
\text { los que no fue } \\
\text { verificable la } \\
\text { observación }\end{array}$ & $\begin{array}{c}\text { Proporción } \\
\text { de estudios } \\
\text { supervisados } \\
\text { con observación } \\
\text { n/N (\%) }\end{array}$ \\
\hline $\begin{array}{l}\text { Relacionadas al contrato } \\
\text { del estudio }\end{array}$ & 8 & $62 / 74(83,8)$ \\
$\begin{array}{l}\text { Observaciones relacionadas } \\
\text { al pago de overhead }\end{array}$ & 2 & $47 / 82(57,3)$ \\
$\begin{array}{l}\text { Documentos regulatorios } \\
\text { (CEI, INS, EsSalud) } \\
\text { incompletos }\end{array}$ & 0 & $39 / 82(47,6)$ \\
$\begin{array}{l}\text { Registros de calibración no } \\
\text { actualizados }\end{array}$ & 1 & $11 / 81(13,6)$ \\
$\begin{array}{l}\text { Documentos del equipo de } \\
\text { investigación incompletos } \\
\text { (CV, BPC) }\end{array}$ & 0 & $11 / 82(13,4)$ \\
$\begin{array}{l}\text { No contar con todos } \\
\text { los equipos e insumos } \\
\text { necesarios para } \\
\text { cumplimiento de normas de } \\
\text { bioseguridad }\end{array}$ & 10 & $9 / 72(12,5)$ \\
$\begin{array}{l}\text { No tener disponible copia } \\
\text { del contrato }\end{array}$ & 0 & $8 / 82(9,8)$ \\
$\begin{array}{l}\text { Exámenes no pagados a la } \\
\text { institución }\end{array}$ & 0 & $5 / 82(6,1)$ \\
\hline
\end{tabular}

CEI: Comité de Ética en Investigación; INS: Instituto Nacional de Salud; $\mathrm{CV}$ : currículum vitae; BPC: buenas prácticas clínicas.

Se observó una disminución en el número de estudios supervisados que no realizaron el pago por concepto de overhead de $72 \%$ en los estudios supervisados en el 2016 a $36 \%$ en el 2017 , lo que estaría relacionado con la implementación de supervisiones de EC en EsSalud. Considerando que en EsSalud existe normativa específica sobre el tema ${ }^{(15)}$, se espera encontrar cada vez menos observaciones al respecto.

Las observaciones relacionadas a documentos; específicamente no tener disponibles todos los documentos regulatorios, incluyendo aprobaciones y comunicaciones con los comités de ética, Instituto Nacional de Salud (INS) y EsSalud fueron las terceras en frecuencia (48\%). Lo reportado en el presente estudio es coincidente con lo encontrado por Gobel et al. en un estudio realizado en colaboración con 15 compañías farmacéuticas, en el cual las observaciones encontradas con mayor frecuencia en las inspecciones de EC en Alemania y otros países de Europa estuvieron relacionada a la documentación ${ }^{(5)}$.

Otros estudios no han reportado de forma específica las observaciones relacionadas a bioseguridad o calibración de equipos, pero estas podrían haber sido incluidas en otras categorías generales como «seguridad» o «aseguramiento de la calidad» ${ }^{(5,6)}$. El presente estudio encontró que hubo reducción en las observaciones relacionadas a bioseguridad, de $16 \%$ en el 2016 a ninguna en el 2017 y 2018, posiblemente por la supervisión constante que se realiza. En los hospitales donde se cuenta con un único centro de investigación, no hubo observaciones relacionadas a bioseguridad ni a la calibración de equipos. El contar con un único centro, en lugar de varios, podría facilitar la implementación de medidas de bioseguridad y la gestión relacionada a la calibración de equipos.

La principal limitación de nuestro estudio es la selección por conveniencia de los ensayos clínicos a ser supervisados. Sin embargo, se debe tener en cuenta que fueron seleccionados según criterios de riesgo y se ha incluido todas las supervisiones realizadas durante cuatro años. Como otra limitación, al tratarse de supervisiones que tienen principalmente un enfoque administrativo y regulatorio, no permiten evidenciar otros hallazgos $u$ observaciones que se podrían encontrar en la supervisión realizada por una autoridad reguladora de EC, por un CEI o durante el monitoreo por parte de los patrocinadores. No se ha profundizado la evaluación de historias clínicas, por lo que podrían existir EAS que no hayan sido comunicados ni evidenciados en las supervisiones $u$ otras incongruencias que se podrían detectar sólo con la revisión exhaustiva de historias clínicas. No obstante, en cuanto a los eventos adversos serios, consideramos que la posibilidad que no hayan sido comunicados al CEI ni a la autoridad reguladora, y por lo tanto, no hayan sido evidenciados en las supervisiones es baja.

En conclusión, las supervisiones a EC brindan aportes valiosos para identificar oportunidades de mejora, regular y gestionar la investigación en EsSalud, constituyendo una experiencia que puede ser útil para otras instituciones. La mayoría de estudios supervisados fueron doble ciego, de fase III y patrocinados por la industria farmacéutica. Las principales observaciones encontradas fueron las relacionadas al contrato del estudio, a documentos regulatorios y al pago por concepto de overhead. Así mismo, debemos resaltar la mejora en los indicadores de supervisión a lo largo de estos cuatro años, como pago de overhead o cumplimiento de medidas de bioseguridad; lo cual respalda la necesidad de realizar supervisiones a los EC.

Contribuciones de autoría: JAGM, MC y JA tuvieron la idea del estudio. JAGM, FPR y VAA realizaron la recolección y análisis de los datos. Todos participaron del diseño del estudio y elaboraron el primer borrador del manuscrito e interpretación de los resultados, hicieron contribuciones significativas al manuscrito, aprobaron su versión final y se hacen responsables del contenido.

Fuentes de financiamiento: El presente estudio fue financiado por el Instituto de Evaluación de Tecnologías en Salud e Investigación - IETSI, EsSalud.

Conflictos de interés: Al momento de la concepción del proyecto y redacción del artículo los autores laboraban en el Instituto de Evaluación de Tecnologías en Salud e Investigación - IETSI, EsSalud. 


\section{REFERENCIAS BIBLIOGRÁFICAS}

1. National Heart Lung and Blood Institute (NHLBI). Clinical Trials [Internet]. Bethesda, MD: NIH; c2018 [citado el 7 de diciembre de 2018]. Disponible en: https://www.nhlbi.nih.gov/health-topics/ clinical-trials

2. Instituto de Evaluación de Tecnologías en Salud e Investigación - IETSI. Reglamento de Organización y Funciones [Internet]. Lima: EsSalud; 2015 [citado el 30 de noviembre de 2018]. Disponible en: http://www.essalud.gob.pe/transparencia/ pdf/rof/rof_IETSI.pdf

3. Aprueban Reglamento de Ensayos Clínicos - Decreto Supremo - No 021-2017-SA [Internet]. Diario Oficial del Bicentenario El Peruano. 2017 [citado 2 de noviembre de 2018]. Disponible en: https://busquedas. elperuano.pe/normaslegales/apruebanreglamento-de-ensayos-clinicos-decretosupremo-n-021-2017-sa-1538902-2/

4. Instituto de Evaluación de Tecnologías en Salud e Investigación - IETSI. Resolución $\mathrm{N}^{\circ}$ 021-IETSI-ESSALUD-2016 que aprueba la Directiva $\mathrm{N}^{\circ}$ 04-IETSIESSALUD-2016 "Directiva que establece los lineamientos de desarrollo de la investigación en EsSalud" [Internet]. Lima: EsSalud; 2016 [citado el 15 de junio de 2019]. Disponible en: http://www.essalud. gob.pe/ietsi/pdfs/direcc_invest_salud/ Direc_Des_dela_invest_Direct_regula_ utilizac_Fondo_de_investigacion.pdf

5. Göbel C, Baier D, Ruhfus B, Hundt F. GCP inspections in Germany and Europe following the implementation of the Directive 2001/20/EC. Ger Med Sci. 2009;31(7):1-9. doi: 10.3205/000060
6. Morgan-Linnell SK, Stewart DJ, Kurzrock R. U.S. food and drug administration inspections of clinical investigators: Overview of results from 1977 to 2009 . Clin Cancer Res. 2014;20(13):3364-70. doi: 10.1158/1078-0432.CCR-13-3206

7. Garmendia CA, Epnere K, Bhansali N. Research Deviations in FDA-Regulated Clinical Trials: A Cross-Sectional Analysis of FDA Inspection Citations. Ther Innov Regul Sci 2018;52(5):57991. doi: 10.1177/2168479017751405.

8. Seguro Social de Salud del Perú. Estadística Institucional [Internet]. Lima: EsSalud; 2019 [citado el 30 de abril de 2019]. Disponible en: www.essalud. gob.pe/estadistica-institucional/

9. Instituto Nacional de Salud. Registro Peruano de Ensayos Clínicos - Búsqueda de Ensayos Clínicos [Internet]. Lima, Perú: INS; 2018 [citado el 30 de noviembre de 2018]. Disponible en: https://ensayosclinicos-repec.ins. gob.pe/acerca-del-repec/busqueda-deensayos-clinicos

10. U.S. National Library of Medicine. Home - ClinicalTrials.gov [Internet]. Bethesda, MD: NLM; c2018 [citado el 30 de noviembre de 2018]. Disponible en: https://clinicaltrials.gov/

11. Minaya G, Fuentes D, Obregón C, AyalaQuintanilla B, Yagui M. Características de los ensayos clínicos autorizados en el Perú, 1995-2012. Rev Peru Med Exp Salud Publica. 2012;29(4):431-6.

12. Marwah R, Van de Voorde K, Parchman J. Good clinical practice regulatory inspections: Lessons for Indian investigator sites. Perspect Clin Res. 2010;1(4):151-5. doi: 10.4103/22293485.96453

13. Mahajan P, D'Souza N, Bhatt A, Halbe V, Sharma R, Narayanswamy S, et al. US food and drug administration Indian site inspections: An experience. Perspect Clin Res. 2012;3(2):73-9. doi: 10.4103/2229-3485.96453

14. Asada R, Yoshimura K, Hattori K, Nonaka Y, Kasai H, Shimizu S. A descriptive research: Exclusion from submitted clinical data package in the review process of new drug approval due to GCP violation in Japan. Contemp Clin Trials Commun. 2019;15:100416. doi: 10.1016/j.conctc.2019.100416

15. Instituto de Evaluación de Tecnologías en Salud e Investigación - IETSI. Resolución $\mathrm{N}^{\circ}$ 05-IETSI-ESSALUD-2016 que aprueba la Directiva $\mathrm{N}^{\circ}$ 01-IETSIESSALUD-2016 "Directiva que regula la utilización del fondo para el desarrollo de la investigación en salud - EsSalud" [Internet]. Lima: EsSalud; 2016[citado el 30 de noviembre de 2018]. Disponible en: http://www.essalud.gob.pe/ietsi/ pdfs/direcc_invest_salud/RIETSI_05_ ESSALUD_2016.pdf

Correspondencia: Jorge A. Garcia-Mostajo Dirección: Av. Arenales 1302, Oficina 310 Jesús Maria, Lima, Perú Teléfono: +5112656000 Anexo 1955 Correo electrónico: jalonsogm@hotmail.com 